\title{
Influence of infrared radiation on the electrical characteristics of the surface-barrier nanostructures based on MBE $\mathrm{HgCdTe}$
}

\author{
Malgorzata Pociask-Bialy ${ }^{1, *}$, Ihor Izhnin ${ }^{2,3}$, Alexander Voitsekhovskii ${ }^{3,4}$, Sergey Nesmelov ${ }^{3,4}$, and Stanislav Dzyadukh ${ }^{3,4}$ \\ ${ }^{1}$ Faculty of Mathematics and Natural Sciences, University of Rzeszow, 35-310 Rzeszow, Poland \\ ${ }^{2}$ Scientific Research Company "Carat", 79031 Lviv, Ukraine \\ ${ }^{3}$ National Research Tomsk State University, 634050 Tomsk, Russia \\ ${ }^{4}$ Siberian Physical-Technical Institute TSU, 634050 Tomsk, Russia
}

\begin{abstract}
Impact of illumination on the admittance of the MIS structures based on $\mathrm{MBE}^{\mathrm{Hg}} \mathrm{g}_{1-\mathrm{x}} \mathrm{Cd}_{\mathrm{x}} \mathrm{Te}$ with graded-gap layers and single quantum wells was investigated. It is shown that for $\mathrm{HgCdTe}$-based nanostructures the illumination greatly affects the capacitance and conductance dependencies. The capacitance-voltage characteristics exhibit a low-frequency behavior, which is associated with a decrease in the differential resistance of the space charge region. Especially informative illumination exposure is in the study of deep traps in $n$-HgCdTe $(x=0.21-0.23)$ without graded-gap layer. Illumination leads to the lowfrequency behavior of capacitance-voltage characteristics of MIS structures based on $p$-HgCdTe with $\mathrm{HgTe}$ single quantum well in the active region, and maximums in the voltage dependences do not appear.
\end{abstract}

\section{Introduction}

Nanostructures based on $\mathrm{MBE} \mathrm{Hg}_{1-\mathrm{x}} \mathrm{Cd}_{\mathrm{x}} \mathrm{Te}$ are promise for a new generation of devices for optoelectronics and nanophotonics [1]. Interest in MIS structures based on the HgCdTe is need to passivate the developed devices surface. The usually used method for studying of the MIS structure is a measurement of admittance in various conditions. Additional information on the physical processes in structures can give admittance measurement at illumination $[2,3]$.

This work presents the first results of investigation of the admittance at illumination impact for MIS structures based on $\mathrm{HgCdTe}$ with nanoinclusions in the active region (graded-gap layers, single quantum wells).

\section{Samples and methods}

MIS structures have been prepared on the basis of $n$ $\mathrm{Hg}_{0.78} \mathrm{Cd}_{0.22} \mathrm{Te}$ grown by MBE on the $\mathrm{Si}$ (013) substrates. During growth of the heterostructures, graded-gap layers with high CdTe content were created on both sides of the working layer (figure 1). Before the formation of the insulator coatings, the conductivity and concentration and mobility of majority charge carriers were investigated by Hall measurements at $78 \mathrm{~K}$. We investigated the MIS structures based on $n-\mathrm{Hg}_{0.78} \mathrm{Cd}_{0.22} \mathrm{Te} / \mathrm{Al}_{2} \mathrm{O}_{3}$ with nearsurface graded-gap layer (structure No.1) and without graded-gap layer (structure No.2). The average electron concentration in the epitaxial film was $5.4 \times 10^{14} \mathrm{~cm}^{-3}$ and electron mobility was $34000 \mathrm{~cm}^{2} /(\mathrm{V} \times \mathrm{c})$. For the structure No. 2, the near-surface graded-gap layer was removed by etching in the $\mathrm{Br}_{2}-\mathrm{HBr}$ solution and then, a $\mathrm{Al}_{2} \mathrm{O}_{3}$ insulator was deposited used by plasma enhanced atomic layer deposition [4]. For the structure No. 1, the insulator coating was formed over the graded-gap layer. The study of these samples allows to investigate the influence of graded-gap layers on the properties of MIS structures.

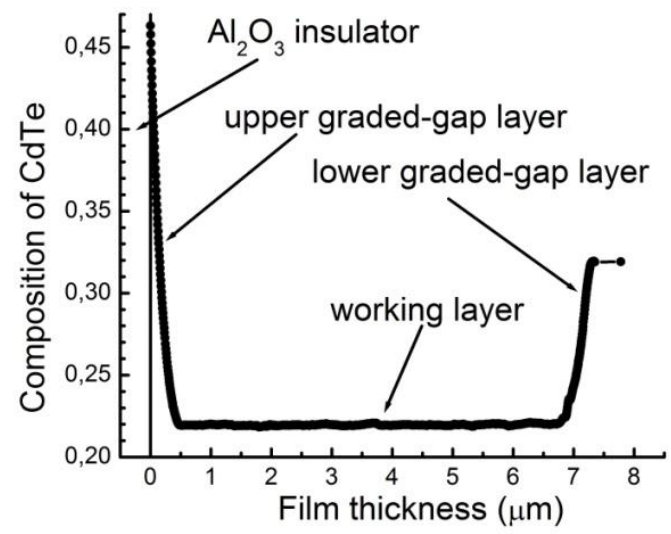

Fig. 1. Distribution of the composition over the thickness of the structure No. 1 based on $\mathrm{MBE} \mathrm{Hg}_{1-x} \mathrm{Cd}_{x} \mathrm{Te}$ measured with an automatic ellipsometer during the growth process.

The samples with HgTe single quantum well (SQW) were fabricated by MBE method based on $p$-HgCdTe on the GaAs (013) substrates at the Institute of Semiconductor Physics of SB RAS (Novosibirsk, Russia). The SQW thickness was $5.6 \mathrm{~nm}$. $\mathrm{Hg}_{0.35} \mathrm{Cd}_{0.65} \mathrm{Te}$ barrier

Corresponding author: i.izhnin@carat.electron.ua 
layers had a thickness of $35 \mathrm{~nm}$. A double-layer insulator $\mathrm{SiO}_{2} / \mathrm{Si}_{3} \mathrm{~N}_{4}$ was deposited over the CdTe $40 \mathrm{~nm}$ layer. Figure 2 shows the basic layout of the layers in the structure with SQW.

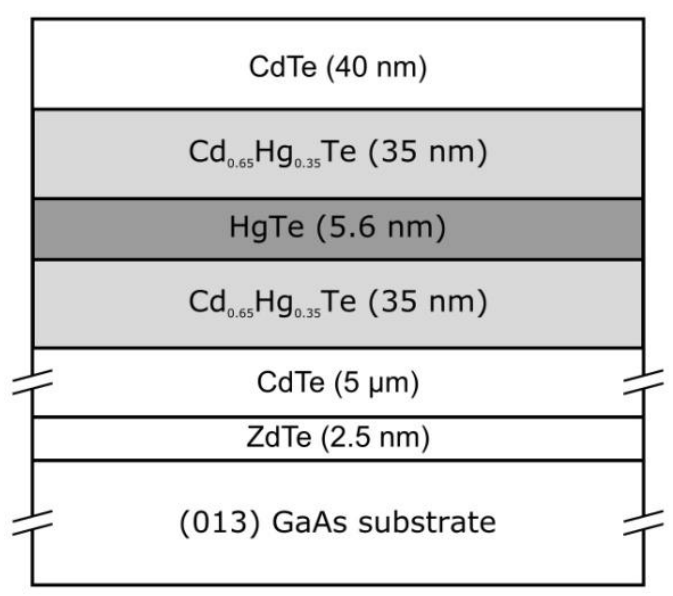

Fig. 2. Scheme of the layers position in structure with SQW.

Measurements were performed with an automated setup for spectroscopy of the nanoheterostructure admittance based on a cryostat Janis and an immittance meter Agilent E4980A. In measurements, the voltage change from negative values to positive ones. Electrophysical characteristics were measured in the dark mode and at illumination through the substrate at light wavelength of 0.94 microns.

\section{Results and discussion}

\subsection{MIS structures based on MBE $n$-HgCdTe with graded-gap layers}

Figure 3 shows the capacitance and conductance versus voltage for MIS structures based on $n-\mathrm{Hg}_{0.78} \mathrm{Cd}_{0.22} \mathrm{Te}$ with (structure No.1) and without (structure No. 2) a gradedgap layer at temperature $15 \mathrm{~K}$ for dark mode and at illumination.

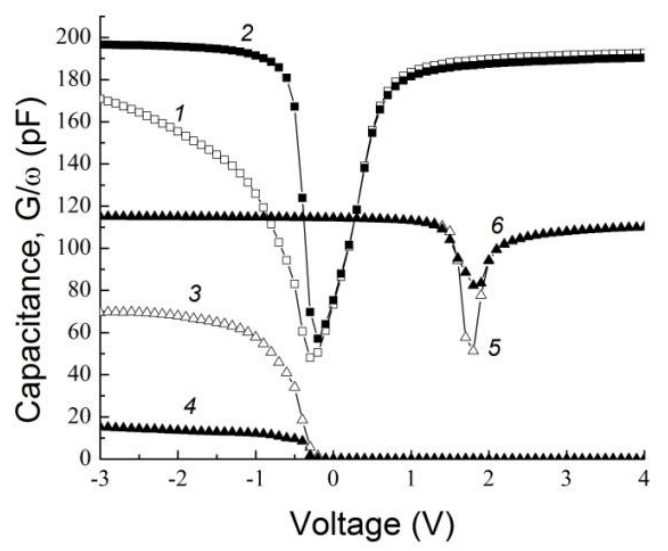

Fig. 3. Voltage dependencies of capacitance (curves 1, 2, 5, 6) and conductance at frequency $20 \mathrm{kHz}$ (curves 3, 4) for MIS structures No. 1 (curves 1-4) and No.2 (curves 5,6) measured at
$15 \mathrm{~K}$ in dark mode (curves $1,3,5$ ) and at illumination (curves 2 , 4, 6). (где ось проводимости?)

Figure 4 shows the values of the equivalent circuit elements of the MIS structure in strong inversion determined from experimental data by the method described in [5]. From Figure 4 can be seen that the capacitance of the inversion layer under illumination increases, due to the increase in the concentration of minority carriers of charge. The illumination reduces the differential resistance of the space charge region (SCR) due to the appearance of an additional mechanism of generation of minority carriers (photogeneration). This reduces the time constant recharging of the inversion layer. The capacitance of the SCR under illumination is somewhat increased, due to the decrease in the maximum thickness of the SCR due to the increase in the concentration of minority carriers in the inversion layer.

From the analysis of Fig. 4, it can be concluded that a illumination strongly changes the capacitance-voltage (CV) characteristics of MIS structures based on $\mathrm{Hg}_{0.78} \mathrm{Cd}_{0.22} \mathrm{Te}$ with a near-surface graded-gap layer. $\mathrm{CV}$ curves at illumination demonstrates a more low-frequency behavior than in the dark mode, and when illuminated capacitance increases in the minimum CV curves. At low frequencies, the illumination decreases the conductance of MIS structures based on $\mathrm{HgCdTe}$ with graded gap layer, and at high frequencies - to increase conductance. For structure No. 2 without the graded-gap layer the impact of illumination is less pronounced, as the $\mathrm{CV}$ curves exhibits

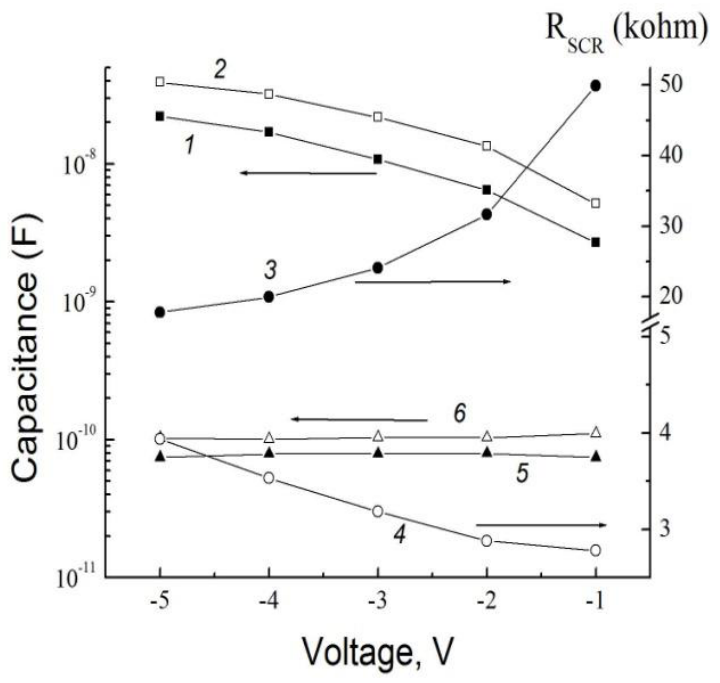

low-frequency behavior of in the dark mode.

Fig. 4. Voltage dependencies of inversion layer capacitance (curves 1,2), resistance of SCR $R_{\mathrm{SCR}}$ (curves 3,4) and capacitance of SCR (curves 5, 6) for MIS structure No. 1 measured at temperature of $15 \mathrm{~K}$ at frequency range of 20-50 $\mathrm{kHz}$ in dark mode (curves 1, 3,5) and at illumination (curves 2, $4,6)$.

In order to calculate the ideal CV-characteristics at illumination, the Poisson equation for a given distribution of CdTe in the near-surface graded-gap layer was solved numerically [6]. In the calculations, we assumed local electroneutrality; the equation was solved taking into

\footnotetext{
Corresponding author: i.izhnin@carat.electron.ua
} 
account the changes in the near-surface graded-gap layer permittivity, band gap, and intrinsic carrier concentration. Account of illumination conducted by adding to the equilibrium concentration of mobile carriers in the expression for the density of the charge carrier concentration encountered during photogeneration.

Figure 5 shows the dependence of semiconductor capacity in the minimum low-frequency $\mathrm{CV}$ characteristics calculated at different temperatures for a structure with graded gap layer and without the graded-gap layer, and the dependence of semiconductor capacity at high frequency in the strong inversion mode calculated for structures with graded-gap layer at a temperature of $77 \mathrm{~K}$.

Convenient to use for the characterization of illumination intensity ratio $\mathrm{p} 1 / \mathrm{p} 0$, where $\mathrm{p} 1$ - concentration of minority carriers under illumination, $\mathrm{p} 0$ - concentration of minority carriers in the dark mode in the semiconductor bulk [3]. It can be seen that for a structure without a graded-gap layer capacity increase in the minimum $\mathrm{CV}$ characteristics begins with small values $\mathrm{p} 1 / \mathrm{p} 0$ (curve 5), for structures with graded gap layer at low values of $\mathrm{p} 1 / \mathrm{p} 0$ capacity at the minimum low-frequency $\mathrm{CV}$ curves does not change, and the increase in capacity at the minimum CV characteristics occurs, starting with a certain value $\mathrm{p} 1 / \mathrm{p} 0$, which increases with decreasing temperature. The reason for this is that the value of $\mathrm{p} 0$, calculated near the interface at the maximum content of CdTe is less than the mean effective concentrations of minority carriers in the SCR as the equilibrium dark concentration of minority carriers in the graded-gap layer increases with distance from semiconductor boundary.

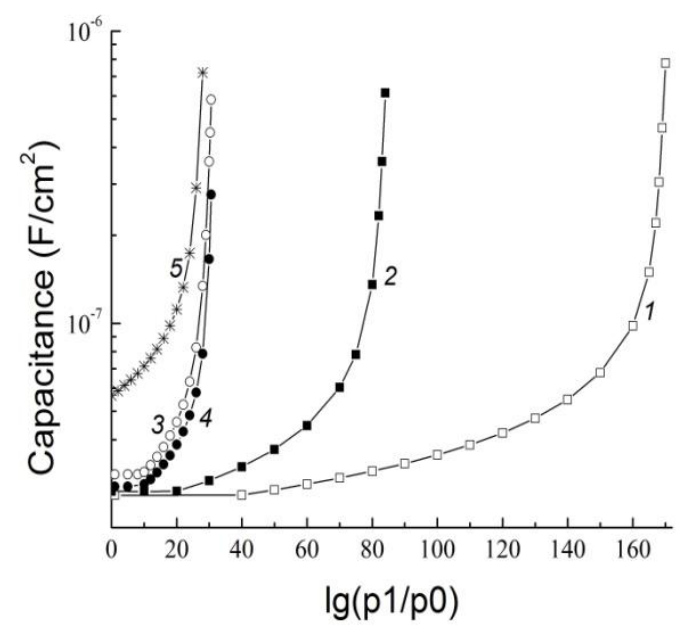

Fig. 5. Calculated dependencies of capacitance in minimum of low-frequency $\mathrm{CV}$ curves versus relation of minority carrier concentration at illumination (p1) to minority carrier concentration in dark mode for graded-gap $n-\mathrm{Hg}_{0.78} \mathrm{Cd}_{0.22} \mathrm{Te}$ at electron concentration of $1.7 * 10^{15} \mathrm{~cm}^{-3}$ for various temperatures 15 (curve 1), 30 (curve 2), $77 \mathrm{~K}$ (curve 3 ), for $n-\mathrm{Hg}_{0.78} \mathrm{Cd}_{0.22} \mathrm{Te}$ without graded-gap layer at $15 \mathrm{~K}$ (curve 5) and high-frequency capacitance in strong inversion mode for graded-gap $n$ $\mathrm{Hg}_{0.78} \mathrm{Cd}_{0.22} \mathrm{Te}$ at $77 \mathrm{~K}$ (curve 4 ).

Investigation admittance under illumination for MIS structures based on $n$ - $\mathrm{HgCdTe}$ in the strong inversion mode allows you to determine the value of the insulator capacitance, which is impossible for MIS structures based on $n-\mathrm{HgCdTe}$ in the accumulation mode due to the effects of degeneracy and non-parabolicity of conduction band.

\subsection{MIS structures based on MBE $n-H g C d T e$ with deep traps}

Especially informative illumination exposure in the study of deep traps in $n$-HgCdTe without graded-gap layer. The appearance of deep traps in the CV characteristics under illumination only was observed (in dark mode, the traps levels are filled, and traps are not manifested in the measurements). If the deep traps appear in dark characteristics, the illumination typically reduces recharge time for deep traps which exhibit a low-frequency behavior at higher frequencies.

It is of interest that the special features associated with the capacity due to a deep level appear in the capacitancevoltage and volt-siemens characteristics of the MIS structures, for which the maximal photo-emf drop is observed under illumination in the strong inversion mode [7]. Without illumination, no special features were observed in the capacitance-voltage characteristics of some samples. Figure 6 shows the CV curves for the MIS $\mathrm{HgCdTe} / \mathrm{AOF}$ structures without a graded-band-gap layer in the dark and on illumination. The capacitance-voltage characteristics for the illuminated MIS $n$-HgCdTe structures with different insulator coatings are found to differ from each other indicating that this deep level is rather due to the epitaxial-film properties than to the insulator-semiconductor interface. The special features in the voltage dependences of capacitance due to the deep level testify to the fact that the states located in the vicinity of the insulator-semiconductor interface (possible, at the interface between the natural oxide layer and epitaxial film) take part in the formation of capacitance properties of the structures. No special features were observed in the capacitance-voltage characteristics under illumination of

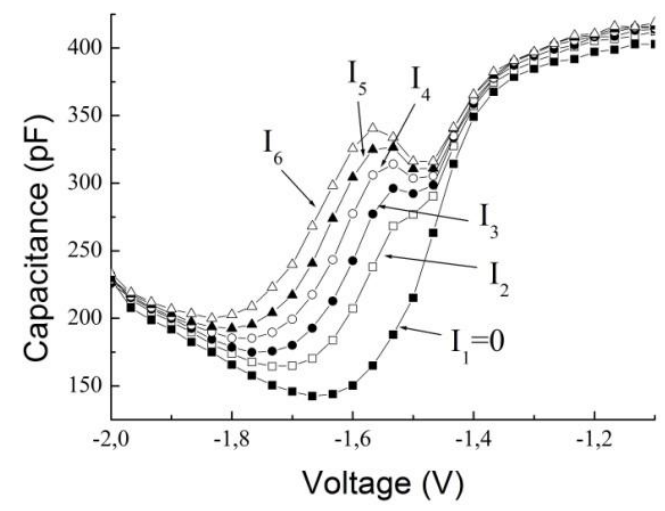

samples produced from other wafers.

Fig. 6. CV curves for MIS structure based on $n-\mathrm{Hg}_{0.77} \mathrm{Cd}_{0.23} \mathrm{Te}$ without graded-gap layer at $77 \mathrm{~K}$ at various intensity of illumination $\left(\mathrm{I}_{6}>\mathrm{I}_{5}>\mathrm{I}_{4}>\mathrm{I}_{3}>\mathrm{I}_{2}>\mathrm{I}_{1}=0\right)$.

Estimations of the deep-level energy position made by the method of high-frequency capacitance-voltage characteristics using the dark capacitance-voltage 
characteristic show that the observed deep level is $0.04-$ $0.05 \mathrm{eV}$ above the valence-band top. The level of a doubly ionized mercury vacancy, being an acceptor center, can have a similar energy position.

Thus, a levels can be present in the near-surface layer of the $n-\mathrm{HgCdTe}$ film. These levels determine the shape of the capacitance-voltage characteristics of the MIS structures under illumination.

\subsection{MIS structures based on MBE $p-\mathrm{HgCdTe}$ with HgTe SQW}

Figures 7 and 8 show dependencies of capacitance on voltage for MIS structure with SQW measured at a temperature of $8 \mathrm{~K}$ for various frequencies of test signal in dark mode and under illumination, respectively. It may be seen that $\mathrm{HgCdTe}$ has a $p$-type conductivity, and in the strong inversion mode at frequencies from $(1-20) \mathrm{kHz}$ non-monotonic change in capacitance is observed due to the recharging quantization levels [8]. At low frequencies $\mathrm{CV}$ characteristic have a low-frequency behavior and maxima become less pronounced than at frequencies of $1-$ $20 \mathrm{kHz}$.

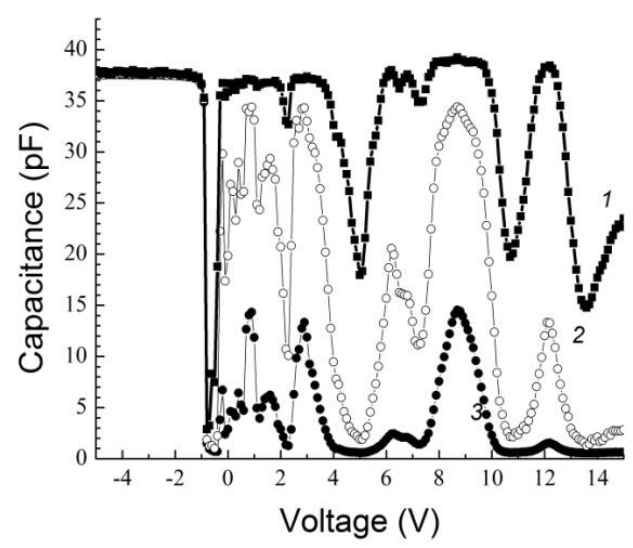

Fig.7. $\mathrm{CV}$ curves for MIS structures based on $p-\mathrm{Hg}_{0.35} \mathrm{Cd}_{0.65} \mathrm{Te}$ with $\mathrm{HgTe} \mathrm{SQW}$ measured at $8 \mathrm{~K}$ in dark mode at various frequencies, kHz: 1 - curve 1,5 - curve 2, 20 - curve 3 .

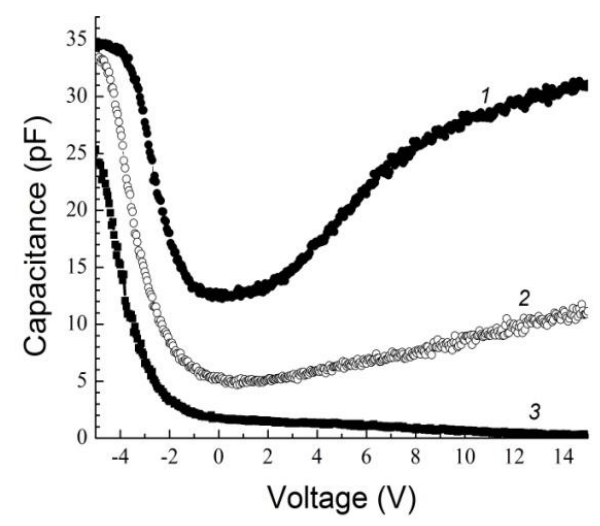

Fig.8. C-V curves for MIS structures based on $p-\mathrm{Hg}_{0.35} \mathrm{Cd}_{0.65} \mathrm{Te}$ with $\mathrm{HgTe} \mathrm{SQW}$ measured at $8 \mathrm{~K}$ at illumination at various frequencies, kHz: 1 - curve 1,5 - curve 2, 20 - curve 3 .
Under illumination maxima in the voltage dependences do not appear (Fig. 8). A possible cause is the photoexcitation of charge carriers from the quantum-well levels.

\section{Conclusions}

Thus, to study the effect of illumination on the electrical characteristics of MIS structures based on $\mathrm{HgCdTe}$, including for cases presence of graded-gap layers and quantum wells in the active region.

$\mathrm{CV}$ measurements have shown that for structure with graded-gap layer under illumination reduces the time of formation of the inversion layer and an increase in capacitance value at the minimum low-frequency $\mathrm{CV}$ curves. Parallel differential conductance of MIS structure under illumination decreases at low frequencies, but increases at high frequencies. The changes under illumination element values equivalent circuit in strong inversion mode at different bias voltages were investigated. Low-frequency and high-frequency $\mathrm{CV}$ characteristics of MIS structures based on MBE $n$ $\mathrm{Hg}_{0.78} \mathrm{Cd}_{0.22} \mathrm{Te}$ in the dark and under different illumination intensities were numerically simulated. For structures with graded gap layer at low illumination intensities, there is a reduction of the surface potential, but there are no changes in the capacitive characteristics.

It is noted that the deep levels manifest themselves in the capacitance-voltage characteristics upon illumination of the MIS structures based on $n-\mathrm{Hg}_{0.78} \mathrm{Cd}_{0.22} \mathrm{Te}$ without graded-gap layer under study. The deep-level energy according to the admittance measurements is $0.04-0.05 \mathrm{eV}$ of the valence-band top.

It is experimentally shown that for MIS structures based on MBE $p$-HgCdTe the presence of a SQW thickness of $5.6 \mathrm{~nm}$ can lead to the appearance of capacitance-voltage characteristics peaks in the stronginversion mode. Under illumination maxima in the voltage dependences do not appear due to photoexcitation of charge carriers from the SQW levels.

This research was supported by the Russian Foundation for Basic Research (grant p_a No.16-42-700759).

\section{References}

1. A. Rogalski, Infrared detectors, New York: CRC Press, Taylor \& Francis Group, 2011

2. V.G. Litovchenko, Surf. Sci., 1, 3, 291 (1964)

3. J. Grosvalet, C. Lund, IEEE Trans. on Electron, Dev., ED-14, 11, 777 (1967)

4. R. Fu, J. Pattison, Opt. Engin., 51, 104003 (2012)

5. A.V. Voitsekhovskii, S.N. Nesmelov, S.M. Dzyadukh, J. Electron. Mater., 45, 2, 881 (2016)

6. A.V. Voitsekhovskii, S.N. Nesmelov, S.M. Dzyadukh, Thin Solid Films, 522C, 261 (2012)

7. A.V. Voitsekhovskii, S.N. Nesmelov, S.M. Dzyadukh, Thin Solid Films, 551, 92 (2014)

8. I.I. Izhnin, S.N. Nesmelov, S.M. Dzyadukh, A.V. Voitsekhovskii, D.I. Gorn, S.A. Dvoretsky, N.N. Mikhailov, Nanoscale Res. Lett., 11, 1, 53 (2016) 